\title{
LA ORIENTACIÓN EN EL MEDIO NATURAL: APRENDIZAJE UBICUO MEDIANTE EL USO DE TECNOLOGÍA
}

\author{
A ORIENTAÇÃO NO AMBIENTE NATURAL: APRENDIZAGEM UBÍQUA ATRAVÉS \\ DO USO DE TECNOLOGIA
}

\author{
ORIENTEERING IN THE NATURAL ENVIRONMENT: UBIQUITOUS LEARNING \\ THROUGH THE USE OF TECHNOLOGY
}

\begin{abstract}
Vanesa Gallego-Lema*, Juan Alberto Muñoz-Cristóbal*, Higinio Francisco Arribas-Cubero*, Bartolomé Rubia-Avi*
\end{abstract}

\begin{abstract}
Palabras clave: Orientación.

Educación superior.

Tecnología

educacional.

Actividad motora.

Resumen: Nuevos estudios emergen en el área de Educación Física, evidenciando que la tecnología puede potenciar el proceso educativo. Este artículo muestra un proceso formativo de los contenidos de orientación como actividad física en el medio natural con apoyo tecnológico. La investigación permitió analizar, siguiendo una metodología de estudio de caso con 65 estudiantes universitarios y un docente, cómo el aprendizaje ubicuo a través del uso de herramientas tecnológicas (Realidad Aumentada, geolocalización, etc.), apoyó el aprendizaje de la orientación en el medio natural. Entre otros resultados, el aprendizaje ubicuo estimuló el proceso de aprendizaje en distintos espacios físicos y virtuales, mejoró la adquisición de contenidos de orientación, la competencia digital, un aprendizaje en distintos momentos, etc. Aparte de estas potencialidades, también se identificaron problemáticas durante el proceso, como la tensión de que la tecnología no eclipsara el contacto con el medio natural, la ausencia de red, y otras.
\end{abstract}

Palavras chave:

Orientação.

Educação superior.

Tecnologia

educacional.

Atividade motora.

Keywords:

Orientation.

Higher Education.

Educational

technology.

Motor activity.

Resumo: Novos estudos surgem na área de Educação Física evidenciando que a tecnologia pode melhorar o processo educativo. Este artigo mostra um processo dos conteúdos de orientação como atividade física no ambiente natural com apoio tecnológico. A pesquisa permitiu analisar, seguindo uma metodologia de estudo de caso com 65 estudantes universitários e um docente, como a aprendizagem ubíqua através do uso de ferramentas tecnológicas (realidade aumentada, geolocalização, etc.) ajudou a aprendizagem no ambiente natural. Entre outros resultados, a aprendizagem ubíqua estimulou o processo de aprendizagem em diferentes espaços físicos e virtuais, melhorou a aquisição de conteúdos de orientação, a competência digital, a aprendizagem em diferentes momentos, etc. Para além dessas potencialidades, também identificaram-se problemáticas durante o processo, tais como: a tensão de que a tecnologia não fosse contra o contato com o meio ambiente natural, a ausência de rede e outras.

Abstract: New studies on Physical Education are appearing, showing that technology can enhance the educational process. This article describes a technology-supported learning process on orienteering as a physical activity in the natural environment. By using a case study with 65 university students and one teacher, the research allowed us to analyze how ubiquitous learning, through the use of technological tools (Augmented Reality, geolocation, etc.) supported learning of orienteering in the natural environment. Among other findings, ubiquitous learning stimulated the learning process in different physical and virtual spaces, better acquisition of orienteering contents, digital competence, and learning at different times. Apart from these potentialities, some difficulties also emerged during the process, such as concerns about the dangers of technology overshadowing students' connections with the natural environment, lack of internet coverage, and others.
* Universidad de Valladolid. Valladolid, España.

E-mail: nesi@gsic.uva.es; juanmunoz@gsic.uva.es; quico@mpc.uva.es; brubia@pdg.uva.es

Recebido em: 06-03-2017 Aprovado em: 16-05-2017

(c) (1) (8) Licence 


\section{INTRODUCCIÓN}

Tener una buena percepción espacial es ser capaz de situarse, moverse y orientarse en el espacio, así como tener conciencia de las posibles situaciones y representarlas. La educación de la percepción espacial en el niño es básica para su desarrollo intelectual y afectivo, así como para la eficacia de su acción motriz (BLÁZQUEZ; ORTEGA, 1984). Dentro de las directrices para realizar una intervención educativa sobre esta capacidad encontramos la orientación en el espacio, definida como la aptitud para mantener constante la localización del propio cuerpo tanto en función de la posición de los objetos en el espacio, como para situar esos objetos en función de su posición (CASTAÑER; CAMERINO, 1991). Esta capacidad se utiliza de forma continua, tanto en nuestra vida cotidiana como durante la realización de cualquier actividad motriz en el medio natural u otros espacios (SANTOS; MARTÍNEZ, 2006). El tratamiento de este contenido en la formación de maestros/as especialistas en Educación Física es importante al menos en tres vertientes, que se relacionan y muestran una cierta continuidad y necesidad de secuenciación para la futura aplicación en Educación Primaria: la orientación como capacidad perceptivo-motriz (conocimiento del cuerpo y del entorno); la orientación como herramienta para el desplazamiento por el medio natural con seguridad y eficacia; y la orientación como actividad deportiva recreativa.

El aprendizaje más allá del aula es un aspecto especialmente relevante en algunas áreas, como es el caso de la Educación Física. El profesorado lleva a cabo las actividades de orientación en diversos espacios físicos: el aula, el patio, el medio natural próximo y conocido hasta el lejano y desconocido. En la actualidad, esta continuidad de la experiencia de aprendizaje entre distintos espacios educativos puede ser apoyada a través de las Tecnologías de la Información y de la Comunicación (TICs). Los avances tecnológicos permiten que cualquier persona pueda crear e irradiar información de tal manera que el aprendizaje pueda llevarse a cabo en cualquier momento y en cualquier lugar (COPE; KALANTZIS, 2010) produciéndose un aprendizaje ubicuo1, instantáneo e interconectado. El aprendizaje ubicuo favorece la unión de las brechas espaciales y temporales, así como la conexión entre los espacios físicos y virtuales gracias a los dispositivos móviles, los cuales por medio de sus herramientas permiten la comunicación, la geoposición, la Realidad Aumentada y otras funciones (VÁZQUEZ-CANO; SEVILLANO, 2015). Diversas investigaciones estudian el apoyo de la tecnología en espacios más allá del aula, en distintos contextos y áreas de conocimiento, como por ejemplo: el aprendizaje de los ángulos en Matemáticas en Educación Primaria a través de la grabación de cortos vídeos desde casa (COOK; PACHLER; BACHMAIR, 2011); actividades que unen los espacios formales, no formales e informales (MILRAD et al., 2013); juegos con innovaciones tecnológicas de Realidad Aumentada más allá del aula para apoyar contenidos turísticos y de patrimonio cultural (TERNIER et al., 2012); una aplicación móvil con preguntas geolocalizadas que los estudiantes responden en un lugar físico determinado (SANTOS et al., 2011); y actividades tecnológicas ubicuas que apoyan el análisis crítico de los anuncios publicitarios (MUÑOZ-CRISTÓBAL et al., 2014).

Dentro del campo de la Educación Física también se están incorporando herramientas cuyo uso permiten un apoyo a aprendizajes específicos de la materia además del desarrollo de la competencia digital (QUINTERO; JIMÉNEZ; AREA, 2016), como por ejemplo: el conocimiento

1 El aprendizaje ubicuo (en inglés ubiquitous learning/u-learning) es aquel conocimiento que se da en cualquier lugar y momento a través de 
de las técnicas de orientación, en las que un uso de la tecnología ubicua promueve la adquisición de aprendizajes y la comunicación entre estudiantes (LAl et al., 2013); un apoyo a los contenidos de orientación a través del uso de códigos QR que posibilita que los estudiantes obtengan un feedback inmediato y una motivación positiva (CASTRO-LEMUS; GÓMEZ, 2016); codificación en códigos QR de vídeos sobre ejercicios de calentamiento y vuelta a la calma (IZQUIERDO, 2013); soporte a los contenidos de orientación en Educación Primaria a través de Realidad Aumentada (MUÑOZ-CRISTÓBAL et al., 2015); práctica de ejercicios de fuerza a través de códigos QR en Educación Secundaria (MONGUILLOT et al., 2014); así como el aprendizaje de la escalada en rocódromo a través de Realidad Aumentada (KAJASTILA; HOLSTI; HÄMÄLÄINEN, 2016).

Sin embargo, a pesar de que hay experiencias que empiezan a incluir tecnología en actividades de orientación en el medio natural (CASTRO-LEMUS; GÓMEZ, 2016; GONZÁLEZLÁZARO; OLALLA; PÉREZ, 2016), éstas todavía siguen siendo puntuales. A lo que se suma las tensiones que dificultan su incorporación en Educación Física en el Medio Natural (EFMN): escasez de horas de la asignatura; miedo a la pérdida de momentos motrices a favor de la tecnología provocando una disminución del tiempo de práctica motriz; y necesidad de formación tecnológica y metodológica adecuada (PRAT; CAMERINO; COIDURAS, 2013). Por ello, se hacen necesarios trabajos de investigación que exploren los efectos del uso de TICs en experiencias de aprendizaje en el dominio de EFMN, como por ejemplo el contenido de la orientación en la naturaleza.

En este trabajo pretendemos dar respuesta a la pregunta de investigación: ¿Cómo las tecnologías ubicuas apoyan el aprendizaje de la orientación dentro de la asignatura EFMN? Para ello, realizamos un estudio de caso en EFMN en la Universidad analizando la repercusión de las tecnologías en el proceso educativo a lo largo de cuatro actividades de orientación en el curso 2013/2014.

\section{DISEÑO DE LA INVESTIGACIÓN}

Esta investigación se centra en la asignatura Educación Física en el Medio Natural (EFMN), de cuarto curso del Grado de Educación Primaria con mención en Educación Física en la Universidad de Valladolid (España). Esta materia es impartida por un profesor con una experiencia docente de 22 años, llevándose a cabo el proceso educativo con 65 estudiantes. Así, investigadores interdisciplinares (Educación Física, TICs en Educación e Ingenieros) del grupo GSIC-EMIC², exploraron el apoyo que supusieron las tecnologías ubicuas para el aprendizaje de los contenidos de orientación, bajo un paradigma de investigación interpretativo mediante la perspectiva del estudio de caso (STAKE, 2005), intentando acceder al problema de manera contextualizada y particularizada.

Las técnicas e instrumentos de recogida de datos que se utilizaron (Tabla 1) fueron entrevistas, observaciones, cuestionarios, focus groups. También se obtuvieron datos a través de los documentos generados por los participantes, como fueron las memorias de la asignatura realizadas por los estudiantes y el diseño educativo de la asignatura. 
Tabla 1 - Disponible en: < https://www.gsic.uva.es/>

Detalle de las técnicas de recogida de datos utilizadas durante la investigación

\begin{tabular}{ll}
\hline Técnica/acrónimo & Descripción \\
\hline $\begin{array}{l}\text { Observación } \\
\text { [Obs] }\end{array}$ & $\begin{array}{l}\text { Observaciones semi-estructuradas, realizadas por varios investigadores. Los datos recogidos } \\
\text { fueron audio/vídeo, fotografías y notas mediante las app Field Notes Pro y Notas en iPad. }\end{array}$ \\
\hline $\begin{array}{l}\text { Cuestionario } \\
\text { [Cuest] }\end{array}$ & Cuestionarios compuestos de preguntas abiertas y cerradas, creados con Google Drive. \\
\hline Entrevista [Ent] & $\begin{array}{l}\text { Conversaciones con los participantes, semi-estructuradas, grabadas mediante la app Audio } \\
\text { Memos en iPad. }\end{array}$ \\
\hline Focus [Focus] & $\begin{array}{l}\text { Conversaciones grupales cara a cara, semi-estructuradas, grabados mediante la app Audio } \\
\text { Memos en iPad. }\end{array}$ \\
\hline
\end{tabular}

La síntesis de información recogida se analizó con la herramienta CSCL-EREM ${ }^{3}$ (JORRÍN-ABELLÁN; STAKE, 2009), la cual facilitó la interacción de los observadores (entre dos y siete investigadores en cada sesión), las observaciones recogidas y los procesos de triangulación. La Figura 1 muestra el proceso de reducción y asociación de datos seguido, intentando responder, en palabras de Stake (2005), al issue o pregunta de investigación planteada. Con el objeto de abordar los distintos aspectos y poder iluminar el issue, se propusieron dos grandes temas (declaraciones temáticas - DT- ), que son: (DT1) aprendizaje ubicuo en la orientación; y (DT2) proceso de aprendizaje de la orientación. A su vez, se dividió cada DT en una serie de preguntas informativas para ayudar a entender y profundizar en cada una de las declaraciones temáticas. En el proceso de reducción de datos se concretaron siete categorías, una correspondiente con la DT1 (aprendizaje ubicuo), y seis con la DT2 (competencia digital, problemáticas, diseño, metodología, contenidos/objetivos/evaluación, compatibilidad tecnología-EFMN).

Figura 1 - Estructura gráfica del proceso de reducción y asociación de datos

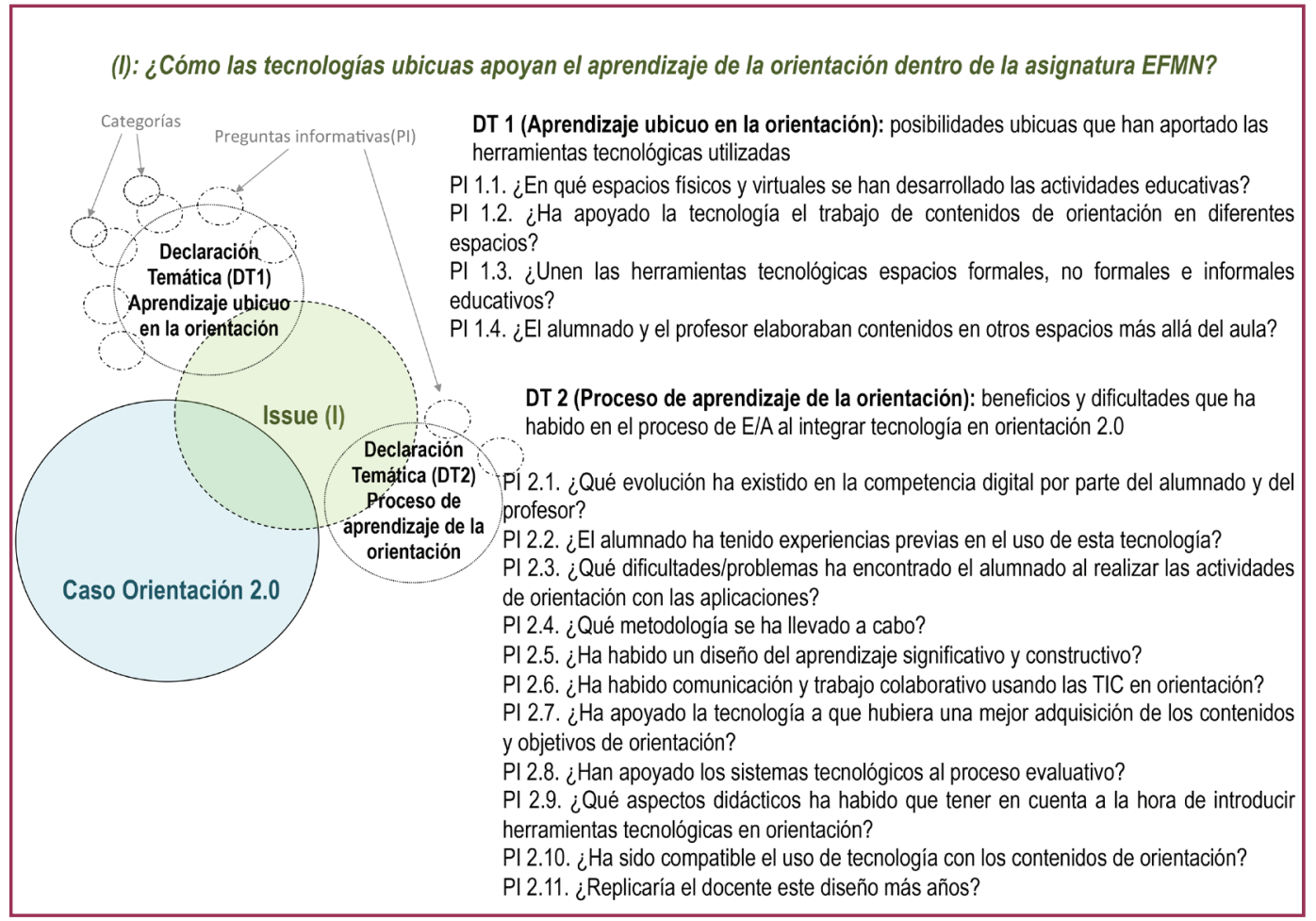

Fuente: Los autores 
La Figura 2 muestra la secuencia de investigación seguida. Durante los meses de agosto y septiembre (Acción 0 -A0- en Figura 2), el docente compartió con los investigadores sus planes educativos anteriores y se diseñó el del futuro curso, comenzando a formarse tecnológicamente con ayuda de un miembro del grupo de investigación. Por otro lado, se usó un cuestionario previo para conocer los antecedentes de alumnado y docente. Al comenzar el curso (A1, A2, A3, A4) se siguió readaptando el diseño educativo y llevándose a cabo las distintas sesiones de EFMN, recogiendo datos de observación de más de 37 horas de actividades de orientación. También era necesario que los estudiantes aportaran sus impresiones y reflexiones, por lo que se realizaron dos focus groups. Al finalizar la materia se preparó otro cuestionario para los estudiantes y se realizaron dos entrevistas al docente y a la investigadora. También se recogieron una serie de documentos: las memorias de la asignatura así como los chats entre los investigadores como medio (in)formativo.

Figura 2 - Acciones, declaraciones temáticas y técnicas de recogida de datos usadas durante la investigación

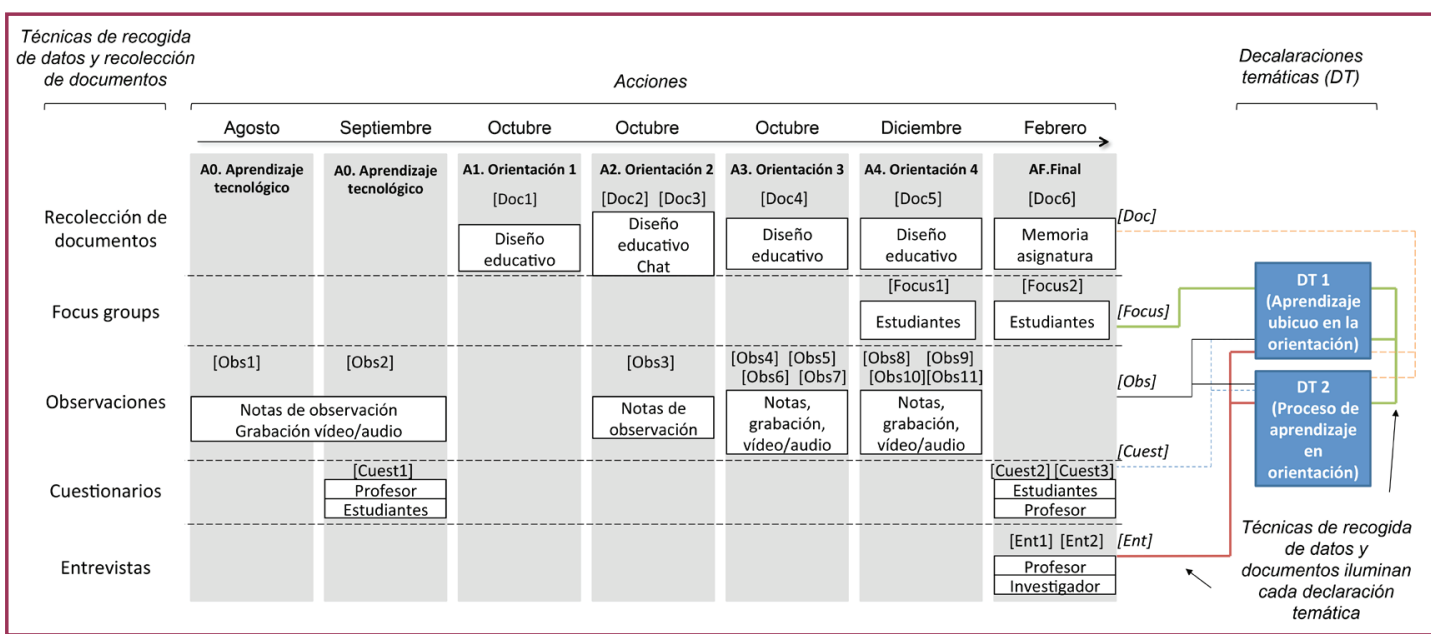

Fuente: Los autores

\section{ORIENTACIÓN 2.0: PROFUNDIZANDO EN EL APRENDIZAJE UBICUO A TRAVÉS DE LAS TICS EN EFMN}

Esta sección describe el proyecto "Orientación 2.0", un conjunto de actividades con implementación tecnológica que apoyaron el aprendizaje de contenidos de orientación dentro de la asignatura EFMN.

Previamente al inicio de la asignatura, docente e investigadores analizaron el diseño educativo para incluir herramientas tecnológicas en EFMN con el objetivo de favorecer la conexión entre distintos espacios, tanto físicos (aula, medio natural, casas, calles, etc.) como virtuales (campus virtual, aplicaciones, etc.) (Figura 3). Esto es posible gracias a la superposición de elementos virtuales en el mundo físico a través de herramientas y aplicaciones específicas de Realidad Aumentada (RA). Dichas TICs fueron:

- Learning Buckets: son contenedores virtuales que agrupan distintas herramientas tecnológicas, tales como páginas webs, Google Docs, etc., embebibles en entornos virtuales de aprendizaje (VLEs, de su traducción al inglés), wikis, etc., apoyando el aprendizaje ubicuo (MUÑOZ-CRISTÓBAL, 2015). Los Learning Buckets permiten posicionar a través de coordenadas geográficas, marcadores de RA, y códigos QR, para posteriormente acceder 
al contenido en distintos espacios -físicos y virtuales- por medio de aplicaciones web y móviles de RA.

- Runkeeper4: es una aplicación móvil que registra el recorrido realizado y aporta información de la actividad deportiva llevada a cabo (tiempo, velocidad, etc.).

- L4C (apple) / C:geo (android): son aplicaciones móviles que se basan en el juego de búsqueda de tesoros (geocachés) al aire libre llamado geocaching ${ }^{7}$.

- Junaio ${ }^{8}$ y Layar9: son navegadores de Realidad Aumentada a través de los cuales se puede ver información geoposicionada.

- Neoreader ${ }^{10}$ : es un lector de códigos QR.

- Google Earth ${ }^{11}$ : es una aplicación que permite visualizar información geográfica en mapas 2D y $3 \mathrm{D}$.

- Facebook, Twitter y Youtube: son aplicaciones basadas en redes sociales que facilitan la información y la comunicación entre personas.

- Line Brush: es una aplicación móvil que permite dibujar nuevos diseños o modificar imágenes.

- Picasa ${ }^{12}$ (actualmente Google Fotos ${ }^{13}$ ): era una aplicación que organizaba y almacenaba las imágenes.

Figura 3 - Representación gráfica de las "capas" virtual y física que permitieron superponer elementos virtuales en los espacios físicos donde se desarrolló la asignatura

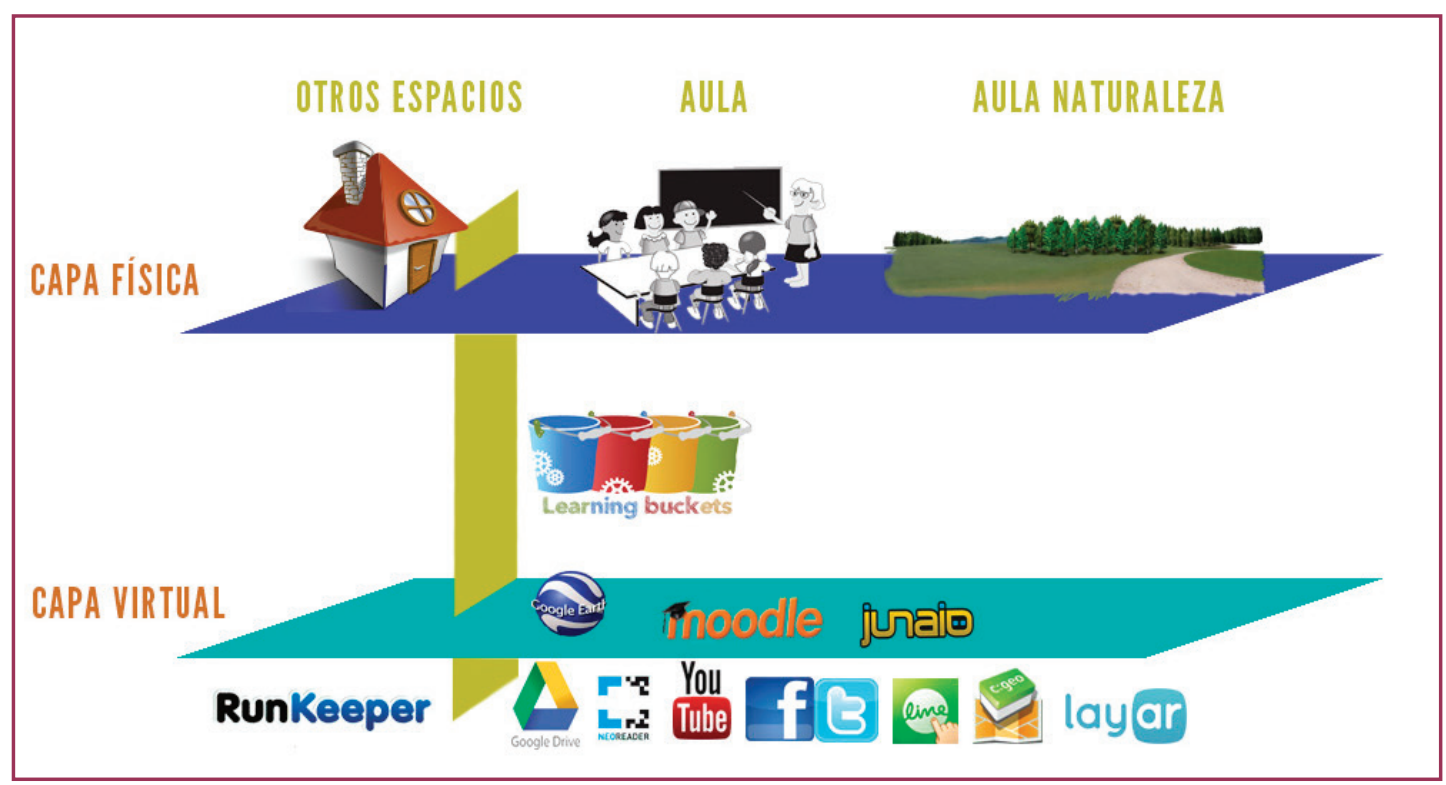

Fuente: Los autores

4 Disponible en: $<$ https: //runkeeper.com/>

5 Disponible en: $<$ https://www.looking4cache.com/>

6 Disponible en: < http://www.cgeo.org/>

7 Disponible en: < https://www.geocaching.com>

8 Disponible en: < https://en.wikipedia.org/wiki/Junaio $>$

9 Disponible en: < https://www.layar.com/>

10 Disponible en: < http://www.neoreader.com/>

11 Disponible en: < http://www.google.es/intt/es/earth/>

12 Aplicación retirada del mercado

13Disponible en: < https://photos.google.com/?hl=es> 
El proyecto Orientación 2.0 se dividió en cuatro sesiones. Durante la primera sesión se sentaron las bases en un espacio conocido para los estudiantes (aula y campus), trabajando el mapa (simbología, escala,...) en grupos/parejas en un circuito de actividades. En las sucesivas sesiones se implementó tecnología para apoyar el aprendizaje, por medio de: aplicaciones para generar dinámicas de trabajo de contenidos, repaso y evaluación; herramientas que conectaran el aprendizaje en distintos momentos y lugares; así como el uso de redes sociales donde los estudiantes pudieran compartir información y contenido. La sesión 2 consistió en una carrera de orientación en un parque urbano donde trabajaron los conceptos de orientación en un espacio más amplio y menos conocido. Algunos de los objetivos fueron: proporcionar recursos esenciales para la utilización del mapa y sus elementos básicos (escala y leyenda); participar en una carrera de orientación y afianzar los contenidos; y manejar herramientas tecnológicas valorando su aplicación a la orientación. La clase se organizó en dos grupos que rotaban en dos actividades simultáneas:

A) La brújula: un grupo trabajaba las partes y utilización de la brújula, realización de rumbos y uso combinado del plano con la brújula.

B) Recorrido de orientación por el parque: el otro grupo, por parejas, realizaba un recorrido de orientación con ocho balizas, tres de las cuales incluían un código QR (Figura 4). Para poder acceder al contenido del código QR utilizaron la aplicación Neoreader. Cada uno de los códigos QR contenían un tipo de actividad: un cuestionario elaborado en Google Drive sobre sus conocimientos de orientación, otro cuestionario de observación del entorno, y finalmente subir a un contenedor (Picasa, en la actualidad Google Fotos) una foto representativa de la jornada. Otras actividades que se llevaron a cabo durante el recorrido fue registrar, con la aplicación móvil Runkeeper, el track realizado. Así, al finalizar la carrera dibujaron sobre el mapa el recorrido que pensaban que habían realizado y lo compararon con el registrado en la aplicación, pudiendo autoevaluarse en el momento y compartirlo con compañeros y profesor.

La sesión 3 se llevó a cabo de manera paralela con otras actividades (piragüismo, senderismo, etc.) en una salida de dos días a un espacio natural lejano (Parque Natural de la Montaña Palentina). El uso de herramientas tecnológicas estaba orientado a proporcionar a los estudiantes un enriquecimiento del aprendizaje y un mayor conocimiento de recursos para trabajar estos contenidos con alumnado de Educación Primaria. El objeto de esta actividad era realizar un recorrido de orientación para encontrar siete balizas (Figura 5), que eran siete blasones de piedra esculpidos en la fachada de edificios históricos. Distribuidos por parejas, los estudiantes utilizaron su mapa de orientación y tablet para hallar las balizas, en las cuales se encontraban distintos tipos de actividades diseñadas, a los que podían acceder de las siguientes formas: (1) a través de un código QR (para leerlo utilizaron la app Neoreader); (2) mediante geolocalización de los contenidos (con aplicaciones de Realidad Aumentada); y (3) a través de marcadores de Realidad Aumentada (dibujos geométricos que eran reconocidos usando aplicaciones de Realidad Aumentada). Las actividades se centraron en responder un cuestionario, averiguar quién/qué era un personaje/elemento famoso del pueblo, o encontrar un geocaché con una aplicación de geocaching. Al finalizar el recorrido de orientación, dibujaron el trayecto que creían haber realizado sobre un mapa descargado en las tablets, a través de la aplicación móvil Line Brush. 
Figura 4 - Relación de balizas y actividades tecnológicas de la sesión 2

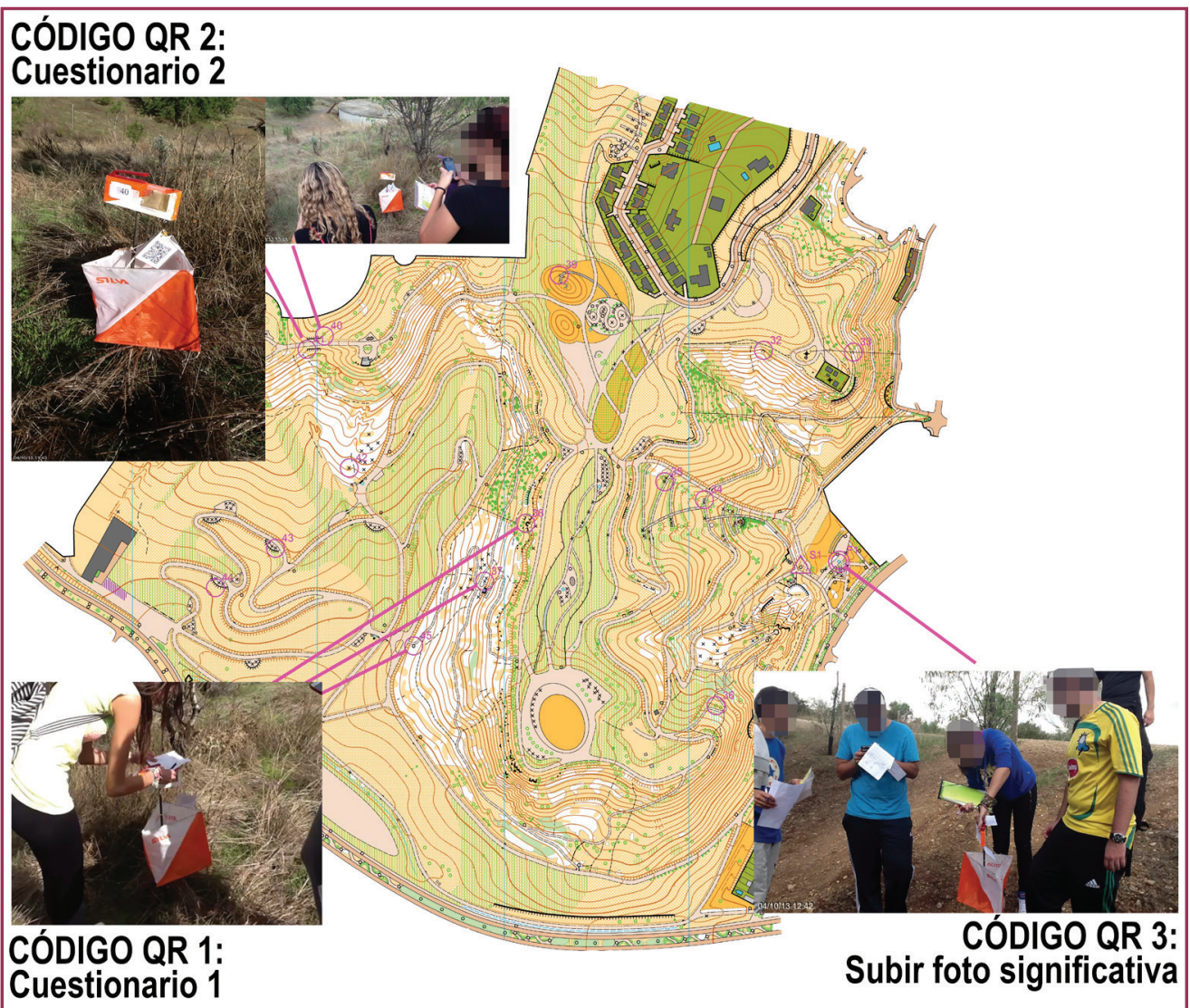

Fuente: Los autores con mapa de orientación cedido por el Club de Orientación Valladolid.

Figura 5 - Recorrido de orientación tecnológico e imágenes del desarrollo de la sesión 3

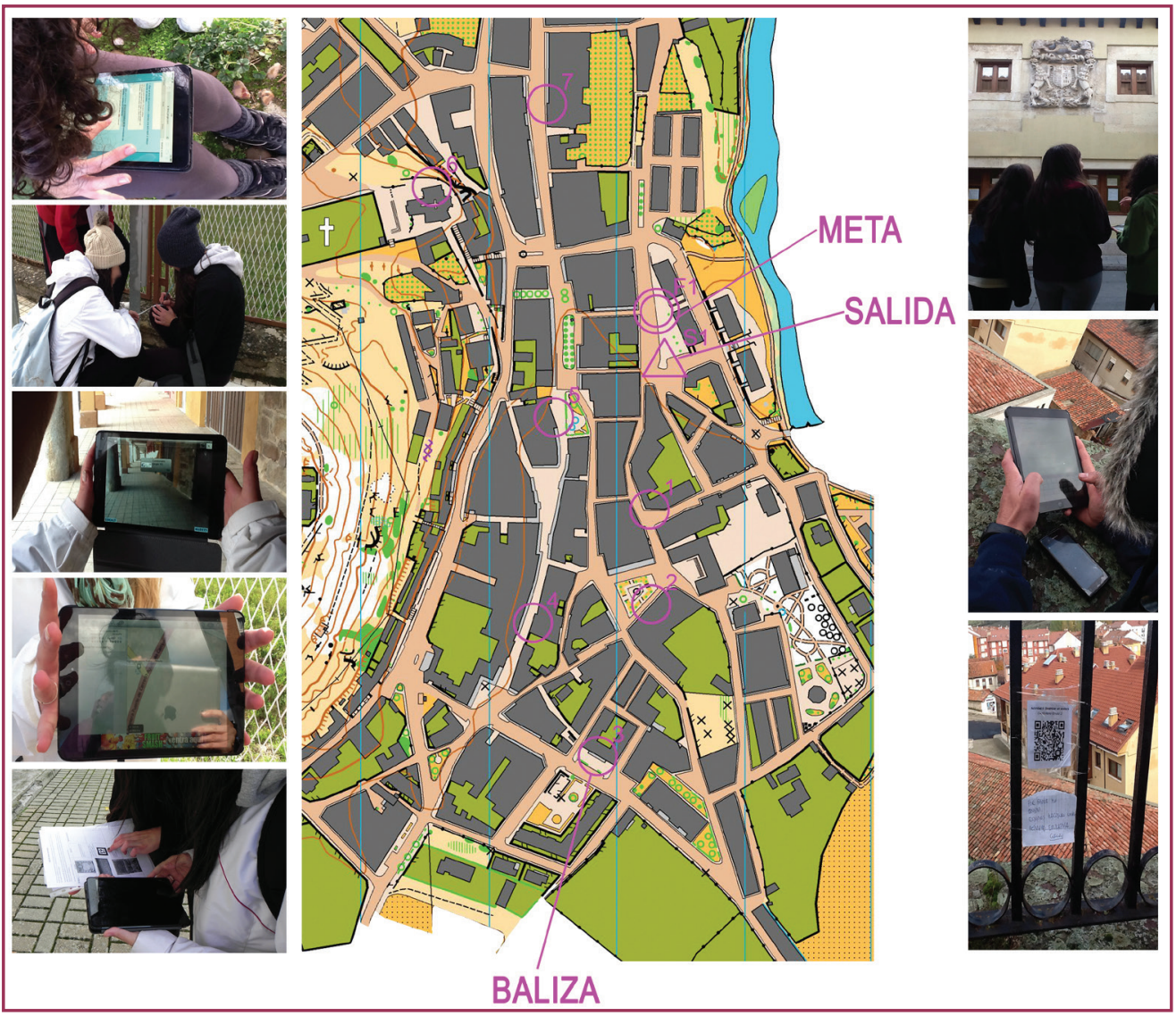

Fuente: Los autores con mapa de orientación cedido por el Club de Orientación Río Carrión. 
Al terminar la asignatura se realizó un gran despliegue de actividades (sesión 4) en el Campus de la Universidad, cuyo objetivo era cubrir todos los contenidos tratados. Así, los estudiantes se dividieron en diez grupos realizando las tareas en siete estaciones. El trabajo de orientación se llevó a cabo a través de las siguientes actividades ${ }^{14 .}$.

A) O-BTT: recorrido de orientación en bicicleta de montaña (BTT) para encontrar dos balizas con códigos $\mathrm{QR}$ que daban acceso a un cuestionario con contenidos relacionados con BTT, y también subir a Picasa una fotografía del lugar donde estaba la baliza.

B) Geocaching: tuvieron que buscar un geocaché próximo al Campus.

C) Orientación con Google Earth: un estudiante de cada grupo hacía un recorrido por el campus con un ipad. Mientras tanto, los compañeros/as que se quedaban en el aula podían ver a través de Google Earth en todo momento su localización en tiempo real, y pintaban en un mapa el recorrido que el compañero/a estaba realizando.

D) O-Walkie: por parejas, un estudiante tenía que encontrar una baliza por el Campus. Para ello, su compañero/a le daba indicaciones de la dirección que tenía que tomar a través de un walkie-talkie. En las balizas se colocó un código QR en el cual los estudiantes contestaban a un cuestionario Google Drive con preguntas relacionadas sobre accesibilidad y orientación.

E) O-Precisión: por parejas, uno en silla de ruedas y el otro caminando, tenían que buscar una baliza en el Campus con las normas de la modalidad inclusiva de orientación O-Precision ${ }^{15}$. Para que los estudiantes conocieran qué tenían que hacer en esta actividad tuvieron que leer un código QR.

\section{RESULTADOS}

En esta sección se describen algunos de los principales resultados obtenidos en el proceso de investigación, organizados según las declaraciones temáticas que definimos en el proceso de reducción de datos (Figura 1).

\section{a) Aprendizaje ubicuo en la orientación}

En este apartado analizamos las posibilidades ubicuas que aportaron las herramientas utilizadas durante el desarrollo de Orientación 2.0, valorando cómo la tecnología puede ayudar a conectar distintos espacios físicos y virtuales. En relación con la capa física, el docente comenzó desarrollando las actividades de orientación desde los entornos más cercanos y conocidos por el alumnado, hasta otros más lejanos y desconocidos. Se utilizaron herramientas que conectaron diversos espacios físicos (aula-casa-calle-medio natural, etc.) con virtuales (redes sociales, herramientas Web 2.0, etc.) mediante una superposición de elementos a través de RA. Esta tecnología facilitó un aprendizaje ubicuo en distintos espacios, favoreciendo: un aprendizaje de los contenidos a través de cuestionarios, a tomar conciencia del entorno estimulando su observación, una evaluación de los estudiantes, la comunicación entre estudiantes y profesor, etc.:

Docente: Me ha sorprendido especialmente como las herramientas tecnológicas elegidas nos han permitido, en tiempo real, la evaluación del profesor y la auto y

14 Disponible en: < https://drive.google.com/open?id=1gHZwlWO6GyL8UcgNud HTiS2YOM\&usp=sharing > . 
coevaluación de los recorridos realizados por los estudiantes, y cómo gracias a la RA se pueden incorporar, en la dinámica de las carreras de orientación, otros aprendizajes. [Ent1]

El profesor diseñó las actividades combinando esta diversidad de espacios, planificando la utilización de herramientas en diferentes lugares y aprovechando el potencial de la geoposición con herramientas tecnológicas para reforzar los aprendizajes "tradicionales" de esta asignatura, tal y como se puede ver en el siguiente extracto:

Docente: de la asignatura hay una cosa muy importante, potente e interesante para trabajar con estos sistemas, porque la geoposición está muy presente y porque el medio natural es un espacio que requiere constantemente del uso de referencias espaciales para un desplazamiento autónomo y seguro. [Obs11]

La implementación tecnológica en Orientación 2.0 apoyó un aprendizaje dentro y fuera del aula de manera ubicua, pudiendo tanto el alumnado como el profesor crear, consultar y realizar actividades de Orientación 2.0, desarrollando por tanto un aprendizaje en cualquier momento y lugar, tal y como exponen Cope y Kalantzis (2010). Este aprendizaje se produjo instantáneamente, ya que el alumnado pudo informarse, comparar, orientarse, autoevaluarse, etc., accediendo a la información y al conocimiento inmediatamente, aspecto que coincide con las aportaciones de Burbules (2012).

Estudiante 2: la tecnología que utilizamos hoy en día, [...] es una forma nueva de educar, yo no tenía ni idea de que existían ese tipo de aplicaciones y que te ayudan a actuar en el momento, no es algo que se vaya a ver cuando se suba, sino que es algo en el momento. [Focus1]

Además, el hecho de que los estudiantes crearan contenidos en distintos espacios provocó que fueran participantes activos del aprendizaje ubicuo generado. El trabajo en distintos espacios suscitó que el alumnado extrapolara el conocimiento a otros ámbitos educativos, del formal al no formal e informal, trasladando esas aplicaciones móviles a otros contextos y conectando los conocimientos académicos con la vida diaria. En este sentido, Bruce (2008) argumenta que quizás el sueño de Dewey se pueda cumplir en la actualidad al unir cuerpo y mente, disciplinas y experiencias ordinarias, tal y como vemos en este estudio. "Estudiante 4: algunos las hemos aplicado a la vida extra-lectiva. Por ejemplo, ayer yo me fui con la bici a hacer una ruta y dije voy a ponerlo a ver que ruta he hecho. [Focus2]."

\section{b) Proceso de aprendizaje de la orientación}

En este apartado profundizamos en los beneficios y dificultades que hubo en el proceso de aprendizaje al integrar la tecnología en los contenidos de orientación. En relación con la competencia digital, el alumnado tuvo pocas experiencias previas y escasa formación universitaria en el uso y aplicación tecnológica de algunas herramientas (Google Maps, Google Drive, redes sociales, Realidad Aumentada y aplicaciones móviles como apoyo para la docencia). El alumnado opinó que, tras finalizar la asignatura, su competencia digital aumentó debido al conocimiento y utilización de una variedad de recursos digitales, tanto para el ocio como para la docencia, reflexionando sobre la importancia de la adquisición de esta competencia de forma transversal desde todas las áreas, así como sobre su futura docencia, tal y como recomiendan Pérez y Vílchez (2013) al sugerir que se aborde esta competencia en las distintas áreas, además de las específicas en TICs, ampliando de esta forma su alcance y relevancia formativa. 
Estudiante 1: he descubierto muchas aplicaciones nuevas que pueden tener cabida en mi futura docencia.

Estudiante 2: hemos conocido muchas nuevas formas de trabajar con estas nuevas tecnologías, tanto iPad como móvil, así como las numerosas aplicaciones hasta ahora desconocidas y que pueden ser de una gran utilidad para nuestro futuro como maestros. [Cuest2]

El docente incrementó sus conocimientos tecnológicos recibiendo apoyo tecnológico por el grupo de investigación hasta llegar a ser autónomo. La evolución como usuario y creador se mostró a lo largo de las sesiones, aconsejando y dirigiendo al alumnado. No obstante, es destacable valorar algún reto pendiente a la hora de sacar más partido al despliegue tecnológico realizado, en relación al tiempo y los recursos empleados, y remarcar también el miedo a sobrecargar de tecnología las actividades desarrolladas en contacto con la naturaleza, perdiendo con ello parte de su esencia.

Docente: he de decir que inicialmente el nuevo enfoque de la asignatura ha supuesto un importante esfuerzo extra en tiempo y esfuerzo, tratando de utilizar siempre la tecnología de forma contextualizada y en su justa medida, [...] para sucesivas puestas en práctica me surgen ideas para aprovechar más aún su potencial, sin perder la pureza que ha de suponer el contacto con la naturaleza, esencial en los aprendizajes de la asignatura. [Ent1]

Hay que mencionar también que los estudiantes y el docente se encontraron con diversos problemas durante las actividades de Orientación 2.0, como fueron: (A) de carácter técnico, como la falta de cobertura, la velocidad de datos, la versión del dispositivo móvil, la batería; (B) relacionados con la actitud y el pensamiento del alumnado, ya que no descargaban las aplicaciones previamente, pasividad y tiempos de espera en el aprendizaje en ciertos momentos, sintieron saturación tecnológica en algunas actividades, y que a algunos estudiantes no les gustó depender de la tecnología en el medio natural; (C) relacionados con el pensamiento del docente, debido al miedo a perder la esencia del contacto con el medio natural a causa de la integración tecnológica, buscando cómo enriquecer el aprendizaje con tecnología sin que ésta eclipsara el medio natural; (D) y de tipo organizativo, como la falta de información tecnológica previa al alumnado, y un gran despliegue de actividades así como el número de estudiantes realizándolas y el tiempo disponible.

En relación con la metodología didáctica, el docente promovió un aprendizaje significativo y constructivo que favoreció la interiorización de los contenidos, como por ejemplo a través de la realización de actividades y cuestionarios a partir de los trabajos previos del alumnado sobre orientación. En relación con esto, Cope y Kalantzis (2010) afirman que el aprendizaje ubicuo ofrece un gran número de posibilidades, como que los estudiantes sean creadores de conocimiento y cultura conectando con sus experiencias y necesidades, así como la posibilidad de trabajar en grupo construyendo un conocimiento colaborativo con tecnología. El hecho de que las actividades con implementación tecnológica fueran colaborativas o no dependió de cómo se diseñara la actividad, del reparto de funciones, del número de dispositivos móviles presentes, etc. El alumnado universitario se repartió las funciones para terminar colaborando por el objetivo, como fue orientarse, contestar a las preguntas, registrar los tracks, ver la información con RA, etc.

El profesor valoró que volvería a llevar a cabo el diseño tecnológico en los siguientes cursos, y aconsejaría a otros docentes que lo realizaran siempre y cuando se les informara 
previamente de la carga de trabajo de planificación que conlleva. De hecho, en la actualidad sigue desarrollando el diseño dentro de la asignatura EFMN aunque ha disminuido la carga tecnológica usada, reflexionando permanentemente sobre los recursos de aprendizaje, ya que no todo es válido para todos los grupos, contenidos, momentos, actividades, etc.

Las evidencias sugieren que la tecnología ubicua favoreció la adquisición de los contenidos de orientación. La tecnología apoyó la geoposición tanto para encontrar lugares como para buscar información. Las herramientas utilizadas posibilitaron compartir rutas en el momento y a través de las redes sociales, registrar itinerarios propiciando una autoevaluación de recorridos, favorecer y apoyar los contenidos de orientación, aumentar la motivación por el aprendizaje, y ampliar el bagaje de recursos tecnológicos e incluirlos tanto en el Practicum y en el Trabajo Fin de Grado como en sus actividades de ocio. El uso de estas herramientas también apoyó el proceso de evaluación, permitiendo la evaluación del profesor así como una autoevaluación y coevaluación de los estudiantes.

Y es que hoy lo decían [los estudiantes] "nos hemos dado cuenta de para qué sirve esto. Que haciendo esta búsqueda tipo yincana, nos hemos dado cuenta de para qué sirve, los contenidos que hemos dado, ...". O sea, que estaban dando una reflexión, de "pues esto tiene una aplicación". [Obs7]

Tras la realización de la asignatura, los beneficios que experimentaron fueron: el conocimiento de nuevas aplicaciones y recursos en el campo educativo, un refuerzo del aprendizaje, motivación, nuevas formas de evaluar, una mayor interiorización y adquisición de los contenidos a través de aprendizajes significativos, un aprendizaje constructivo, un mayor aprendizaje colaborativo y cohesión entre compañeros. El docente manifestó su satisfacción, valorando la tecnología como una gran oportunidad para el área. Así, los objetivos de la asignatura se alcanzaron, uno de ellos referido a la elección y uso de tecnología con la finalidad de enriquecer los contenidos propios de la asignatura.

Docente: los recursos tecnológicos hay que elegirlos cuidadosamente y diferenciar cuándo son ayuda y cuándo estorban para el aprendizaje. [Ent1]

Estudiante: porque son formas de trabajar distintas a las habituales, hecho que conlleva una mayor motivación [...]. Además, experimentar y trabajar con nuevas aplicaciones hasta ahora desconocidas permite desarrollar la competencia digital, teniendo un amplio arco de posibilidades para trabajar con los alumnos en el futuro. [Cuest2]

El docente tuvo en cuenta la compatibilidad entre tecnología y los contenidos de orientación del área de EFMN desarrollando un diseño tecnológico, intentando no perder la esencia del contacto con el medio natural, y teniendo en cuenta que el aprendizaje ubicuo no lo es todo y no todo se puede aprender de esta manera, tal y como afirma Burbules (2012). La clave fue conectar la tecnología con la motricidad, con el medio natural, con la metodología de la asignatura, siendo conscientes de la saturación tecnológica en ciertos momentos y reconsiderando el docente las sucesivas prácticas e intervenciones:

Docente: a veces me sentía culpable de usar los móviles en determinados momentos de la actividad, cuando pensaba que el mirar a la pantalla iba a privarles de disfrutar y aprender con todo lo que le rodeaba [...] el secreto es que nos sirva de apoyo y que nunca eclipse el valor intrínseco de desplazarse por el medio natural. [Ent1] 


\section{CONCLUSIONES}

Los resultados del estudio muestran que las tecnologías ubicuas facilitaron el aprendizaje en distintos espacios y momentos durante el desarrollo de Orientación 2.0. Dichas tecnologías ayudaron a conectar los ámbitos formales, no formales e informales y a que el alumnado participase activamente en su aprendizaje. En este sentido, el aprendizaje ubicuo a través del uso de TICs y de un adecuado diseño educativo, apoyó los contenidos de orientación, la evaluación, los objetivos, y favoreció la mejora de la competencia digital por parte de los estudiantes así como el conocimiento de recursos digitales para la docencia. Además, también apoyó la metodología seguida por el docente, llevándose a cabo un aprendizaje colaborativo cuando la organización de los recursos y de los agrupamientos fue la correcta.

Por el contrario, emergieron limitaciones técnicas, organizativas y otras relacionadas con el pensamiento del alumnado y del profesor durante el desarrollo de la investigación, aspectos que hay que tener en consideración para posteriores intervenciones. A pesar de que se realizaron esfuerzos por no perder la esencia de las actividades en la naturaleza, hubo algunos momentos puntuales en los que la tecnología hizo peligrar el desarrollo de las actividades debido a distintas razones, como por saturación tecnológica o utilización en momentos inadecuados, por no dedicar con antelación tiempo suficiente para explicar el funcionamiento de las aplicaciones, o por problemas técnicos. Por ello, nos parece interesante resaltar la importancia de no caer en un posible desborde tecnológico, buscando el equilibrio entre medio natural, motricidad y tecnología, aspectos que estuvieron presentes en el pensamiento de los estudiantes y del docente.

Aunque el uso de tecnologías ubicuas dentro de Orientación 2.0 no fue el centro del hecho pedagógico, el estudio muestra que éstas apoyaron al campo y engrandecieron los aprendizajes previstos. Creemos que a través del conocimiento de las potencialidades del uso de tecnologías ubicuas en este estudio así como de sus limitaciones, se permite una mejor transferencia de este diseño a otros contenidos de EF, contextos, momentos, etapas educativas, y áreas de conocimiento.

\section{REFERENCIAS}

\section{BLÁZQUEZ, Domingo; ORTEGA, Emilio. La Actividad motriz en el niño de 3 a 6 años.} Madrid: Cincel, 1984.

BRUCE, Bertram C. Ubiquitous learning, ubiquitous computing, and lived experience. In: COPE, Bill; KALANTZIS, Mary. Ubiquitous learning. Urbana: University of Illinois, 2008. p. 21-30.

BURBULES, Nicholas C. Ubiquitous learning and the future of teaching. Encounters on education=Encuentros sobre educación=Recontres sur l'education, v.13, p. 3-14, 2012.

CASTAÑER, Marta; CAMERINO, Oleguer. La educación física en la Enseñanza Primaria: una propuesta curricular para la Reforma. Barcelona: INDE, 1991.

CASTRO-LEMUS, Nuria; GÓMEZ, Iván. Incorporating QR codes in Physical Education in Secondary. Retos: nuevas tendencias en educación física, deporte y recreación, v. 29, p. 114-119, 2016. 
COOK, John; PACHLER, Norbert; BACHMAIR, Ben. Ubiquitous mobility with mobile phones: a cultural ecology for mobile learning. E-Learning and Digital Media, v. 8, n. 3, p. 181-195, 2011.

COPE, Bill; KALANTZIS, Mary. Ubiquitous learning. Urbana: University of Illinois Press, 2010.

GONZÁLEZ-LÁZARO, Javier; OLALLA, David; PÉREZ, María. Aplicación de nuevas tecnologías en la educación física: la carrera de orientación. In: JORNADAS SOBRE EDUCACIÓN FÍSICA EN LA NATURALEZA, 1, 2016. [Anales]... Valsaín (Segovia): REEFNAT, 2016. p. 131-135.

IZQUIERDO, Andrés. Códigos QR flexibles: un proyecto con dispositivos móviles para el trabajo de calentamiento en educación física. EmásF: revista digital de educación física, n. 23, p. 53-71, 2013.

JORRÍN-ABELLÁN, Iván M; STAKE, Robert E. Does ubiquitous learning call for ubiquitous forms of formal evaluation? An Evaluand Oriented Responsive Evaluation Model. Ubiquitous Learning: an International Journal, v. 1, n. 3, p. 71-82, 2009.

KAJASTILA, Raine; HOLSTI, Leo; HÄMÄLÄINEN, Perttu. The Augmented Climbing Wall. In: Conference on Human Factors in Computing Systems'16. New York: ACM, 2016. p. 758769 .

LAl, Hsin-Chih et al. The implementation of mobile learning in outdoor education: application of QR codes. British Journal of Educational Technology, v. 44, n. 2, p. E57-E62, 2013.

MILRAD, Marcelo et al. Seamless Learning: An International Perspective on Next Generation Technology Enhanced Learning. In: BERGE, Zane L; MUILENBURG, Lin Y. Handbook of Mobile Learning. New York: Routledge, 2013. p. 95-108.

MONGUILLOT, Meritxell et al. Una experiencia colaborativa mediante códigos QR. Revista de Universidad y Sociedad del Conocimiento, v. 11, n. 1, p. 175-191, 2014.

MUÑOZ-CRISTÓBAL, Juan A. et al. City Ads: Embedding virtual worlds and Augmented Reality in everyday educational practice. Journal of Universal Computer Science, v. 20, n. 12, p. 1670-1689, 2014.

MUÑOZ-CRISTÓBAL, Juan A. et al. Supporting Teacher Orchestration in Ubiquitous Learning Environments: A Study in K-12 Education. Transactions on Learning Technologies, v. 8, n. 1, p. 83-97, 2015.

MUÑOZ-CRISTÓBAL, Juan A. Supporting teacher ochestration of across-spaces learning situations. 2015. 187 f. Tesis (doctoral). - Universidad de Valladolid, Valladolid , 2015.

PÉREZ, Francisco; VÍLCHEZ, José E. Perception of teachers trainees about the potential of information technology in education: from expectation to reality. Fuentes: Revista de la Facultad de Ciencias de la Educación, n. 13, p. 155-172, 2013.

PRAT, Queralt; CAMERINO, Oleguer; COIDURAS, Jordi. Introducción de las TIC en educación física. Estudio descriptivo sobre la situación actual. Apunts: Educación Física y Deportes, n. 113, p. 37-44, 2013.

QUINTERO, Lucía Esther; JIMÉNEZ, Francisco; AREA, Manuel. Las "e-actividades": aplicaciones y recursos web. Tándem: Didáctica de la educación física, n. 53, p. 12-18, 2016.

SANTOS, María Luisa; MARTíNEZ, Luis Fernando. Propuesta práctica integral para el desarrollo de actividades físicas en el medio natural en el entorno cercano del centro escolar de primaria. 
In: SÁEZ PADILLA, Jesús; SÁENZ-LÓPEZ, Pedro; DÍAZ, Manuel (Org.). Actividades en el medio natural. Huelva: Universidad de Huelva, 2006. p. 65-82.

SANTOS, Patricia et al. QuesTInSitu: From tests to routes for assessment in situ activities. Computers \& Education, v. 57, n. 4, p. 2517-2534, 2011.

STAKE, Robert. Multiple Case Study Analysis. New York: Guilford, 2005.

TERNIER, Stefaan et al. ARLearn: Augmented Reality meets Augmented Virtuality. Journal of Universal Computer Science, v. 18, n. 15, p. 2164, 2012.

VÁZQUEZ-CANO, Esteban; SEVILLANO, María Luisa. Dispositivos digitales móviles en educación: el aprendizaje ubicuo. Madrid: Narcea, 2015. 


\section{Apoyo:}

El trabajo descrito en este artículo ha contado con la financiación parcial del Ministerio de Economía y Competitividad (España), proyecto TIN201453199C32R; Junta de Castilla y León (España), proyecto VA082U16. Los autores también agradecen la colaboración de los estudiantes involucrados en esta investigación, así como el apoyo del grupo de investigación GSIC-EMIC, especialmente a Henry Díaz, Sara Villagrá, Juan I. Asensio y Alejandra Martínez. 